Notfall Rettungsmed 2022 $25: 51-52$ https://doi.org/10.1007/s10049-021-00956-z Angenommen: 21. Oktober 2021 Online publiziert: 12. Januar 2022 (C) The Author(s), under exclusive licence to Springer Medizin Verlag GmbH, ein Teil von Springer Nature 2021

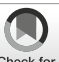

\section{Die „glorreichen Sieben“ der pädiatrischen Notfallmedizin}

\author{
Bernhard Gliwitzky ${ }^{1}$ Florian Hoffmann ${ }^{2}$ - Martin Olivieri' ${ }^{2}$ David Häske ${ }^{1}$ \\ ${ }^{1}$ Sektion Notfall- und Katastrophenmedizin, Deutsche Interdisziplinäre Vereinigung für Intensiv-und \\ Notfallmedizin e.V. (DIVI), Berlin, Deutschland \\ ${ }^{2}$ Sektion Pädiatrische Intensiv- und Notfallmedizin, Deutsche Interdisziplinäre Vereinigung für Intensiv- \\ und Notfallmedizin e. V. (DIVI), Berlin, Deutschland
}

Der pädiatrische Notfall ist mit ca. 5\% der Gesamteinsätze im Rettungsdienst per se selten, weswegen gerade schwer erkrankte und verletzte Kinder für das gesamte Rettungsteam eine große medizinische und emotionale Belastung darstellen. Durch die niedrige Einsatzfrequenz lässt sich außerdem kaum praktische Erfahrung hinsichtlich Patientenbeurteilung und Durchführung spezifischer Maßnahmen erlangen. Trotzdem müssen wir alle Sorge dafür tragen, dass auch das Kind im Notfall eine geeignete und angepasste Versorgung erfährt. Dabei muss der Fokus auf der Patientensicherheit liegen. Keinesfalls dürfen Maßnahmen das Kind weiter schädigen. Daher gilt es gerade im Rettungsdienst immer nach dem Grundsatz zu arbeiten: „So wenig wie möglich, so viel wie notwendig."

\section{》) Die DIVI bietet ein Netzwerk engagierter Notfallsanitäter, Notärzte und Kinderärzte}

Die Sektionen „Notfall- und Katastrophenmedizin" und „Pädiatrische Intensiv- und Notfallmedizin" der Deutschen Interdisziplinären Vereinigung für Intensiv- und Notfallmedizin (DIVI) haben sich zum Ziel gesetzt, den Leserinnen und Lesern der Zeitschrift Notfall+Rettungsmedizin in einer Serie die sieben wichtigsten pädiatrischen Symptomkomplexe und Notfallbilder praxisnah zu präsentieren, damit ein Mehr an Sicherheit bei der Versorgung unserer kleinen Patienten zu bieten und zu entsprechenden Trainings und Fortbildungen zu motivieren. Die sieben Beiträge werden über die nächsten zwei bis drei Jahre in der Rubrik "Teamwork + Education" erscheinen, da sie vor allem den Anspruch haben, auf wesentliche praktische Hilfestellungen zu fokussieren und alle beteiligten Berufsgruppen im Rettungsdienst anzusprechen. Die Artikel werden dabei folgende Themen umfassen und nach und nach publiziert:

- Intranasale Analgesie (diese Ausgabe)

- Luftnot

- Trauma

- Neugeborenenversorgung

- Plötzliche Bewusstseinsstörungen

- Krampfanfall

- Reanimation

Die Autoren werden sich aus der Berufsgruppe der Notfallsanitäter, Notärzte und Pädiater zusammensetzen, um auch immer die verschiedenen Blickwinkel und Perspektiven bei der Versorgung abzubilden. Die DIVI als interdisziplinäre und auch interprofessionelle Fachgesellschaft bietet hier das entsprechende Netzwerk an kompetenten Praktikern aus dem Bereich der Notfallsanitäter, der Notärzte und der Kinderärzte. Wir hoffen sehr, dass Ihnen die Beiträge und auch die damit verbundenen "One Minute Wonder" gefallen. Schreiben Sie uns gerne Ihre Eindrücke zu den Beiträgen.

In dieser Ausgabe der Notfall+Rettungsmedizin erscheint der erste Beitrag zum Thema Analgesie bei Kindern. Wir wünschen viel Spaß bei der Lektüre und dem Erkenntnisgewinn.

Herzlichst

Bernhard Gliwitzky, Florian Hoffmann, Martin Olivieri und David Häske 
Fortbildungstipp

\section{Korrespondenzadresse}

\section{Bernhard Gliwitzky}

Sektion Notfall- und Katastrophenmedizin, Deutsche Interdisziplinäre Vereinigung für Intensiv-und Notfallmedizin e.V. (DIVI) Schumannstraße 2, 10117 Berlin, Deutschland snk@divi.de

Interessenkonflikt. B. Gliwitzky, F. Hoffmann, M. Olivieri und D. Häske geben an, dass kein Interessenkonflikt besteht.



\section{Fragen zur Reanimation von Kindern?}

Ein Kind zu reanimieren ist auch für langjährige Ärtz*innen und Kolleg*innen des Rettungsdienstfachpersonals eine Herausforderungen. Oft stellen sich konkrete Fragen, die einen verunsichern. Lesen Sie hier kurz und knapp die Antworten von PD Dr. Florian Hoffmann auf einige Fragen Ihrer Kolleg*innen und die dazugehörigen Empfehlungen aus der ERC-Reanimationslinein 2021.

Das komplette Webinar zu den ERCReanimationsleitlinen



Im Webinar finden Sie die essentiellen Grundlagen der ERC-Reanimationsleitlinien zusammengefasst. Der Fokus liegt dabei auf Koronarthrombosen und Herzinfarkt und der der Renimation bei Kindern und Jugendlichen.

\section{Die Referenten:}

- Prof. Dr. B. W. Böttiger, Köln

- PD Dr. Florian Hoffman, München

- Dr. med Carsten Lott, Mainz

[URL: www.springermedizin.de/webinar-ercleitlinien]
Antworten aus 3 Fragen zum Webinar:

? Erkennt der AED anhand des Körperwiderstands eine mögliche Reduktion der Energiezahl oder werden bei einem Säugling auch 360J abgegeben?

>> Bisher erkennt der AED nur, ob K-Elektroden oder E-Elektroden angeschlossen sind. In Zukunft wird aber eine Berechnung nach Körperwiderstand realisiert werden können.

? Gibt es eine Empfehlungen zum Zeitpunkt der Intubation unter CPR?

>> Die Intubation ohne Effekt auf Outcome ist sehr in den Hintergrund gerückt und nur dann durchführbar, wenn alle notwendigen Maßnahmen laufen und ein Experte für Kinderintubation anwesend ist ( $>100-150$ Intubationen).

? Gibt es eine Evidenz zur Verwendung von Erwachsenen-Masken bei Kindern, im Sinne einer Full-Face-Alternative, falls kein Kinderbeutel verfügbar ist? >> Es gibt keine Evidenz aber Erfahrungen mit Drehen der Maske um 180 Grad, mit Einmalmasken mit aufblasbarem Rand kann auch mit Erwachsenen-Masken zumeist eine gute Ventilation erreicht werden. 